\title{
Novel Design of Electromagnetic Bandgap Using Fractal Geometry
}

\author{
Huynh Nguyen Bao Phuong, ${ }^{1}$ Dao Ngoc Chien, ${ }^{2}$ and Tran Minh Tuan ${ }^{3}$ \\ ${ }^{1}$ School of Electronics and Telecommunications, Hanoi University of Science and Technology, Hanoi 10000, Vietnam \\ ${ }^{2}$ Department of High Technology, Ministry of Science and Technology, Hanoi 10000, Vietnam \\ ${ }^{3}$ National Institute of Information and Communications Strategy, Ministry of Information and Communications, \\ Hanoi 10000, Vietnam \\ Correspondence should be addressed to Huynh Nguyen Bao Phuong; phuong.huynhnguyenbao@hust.edu.vn
}

Received 7 September 2012; Revised 11 January 2013; Accepted 28 January 2013

Academic Editor: Eric Lheurette

Copyright (C) 2013 Huynh Nguyen Bao Phuong et al. This is an open access article distributed under the Creative Commons Attribution License, which permits unrestricted use, distribution, and reproduction in any medium, provided the original work is properly cited.

\begin{abstract}
A novel electromagnetic bandgap (EBG) structural design based on Fractal geometry is presented in this paper. These Fractals, which are the Sierpinski triangles, are arranged to repeat each $60^{\circ}$ to produce the hexagonal unit cells. By changing the gap between two adjacent Sierpinski triangles inside EBG unit cell, we can produce two EBG structures separately that have broadband and dual bandgap. By using the suspending microtrip method, two arrays $3 \times 4$ of EBG unit cells are utilized to investigate the bandgap of the EBG structures. The EBG operation bandwidth of the broadband structure is about $87 \%$ and of the dual-band structure is about $40 \%$ and $35 \%$ at the center bandgap frequencies, respectively. Moreover, a comparison between the broadband EBG and the conventional mushroom-like EBG has been done. Experimental results of the proposed design show good agreement in comparison with simulation results.
\end{abstract}

\section{Introduction}

Recently, an intensive interest in metamaterials, such as high impedance surface (HIS) composed of metallic or dielectric unit cells, can be observed due to their unique characteristics in antennas and microwave circuits applications [1]. In general, HIS has two unique properties included of electromagnetic bandgap (EBG) and artificial magnetic conductor (AMC). One of the most important properties of EBG structure is to prohibit the propagation of the surface wave $[2,3]$. Practical applications of EBG structure usually have difficulty in accommodating its physical size, because the period of EBG lattices has to be a half-wavelength at the bandgap frequency. This problem had not been solved until the mushroom-like EBG was proposed by Sievenpiper et al. [4]. Then, several of novel EBG structures were presented such as spiral EBG, uniplanar compact EBG (UC-EBG), and fork-like EBG. These structures have several advantages, such as compact size low loss. However, due to their resonant property, all of those structures are narrowband which makes them not practical for use in broadband applications. It is a real challenging task to create an EBG with wide bandwidth.

Several broadband EBG structures were found in the literature. Typically, there have been two approaches aiming to obtain EBG structures with wider bandwidth: the use of EBGs with via-holes [5] (with the inconveniences of complex and expensive manufacturing process) and the adoption of multilayered FSSs over a metallic ground plane or multiperiod mushroom-like structure [6] (which yields less compact designs and is rather expensive). Recent research efforts focus on the development of planar EBG that does not need vias and that can be integrated antenna to enhance the gain and reduce the backward radiation and increasing efficiency [7-9].

As [10], several of Fractal shapes can be used for antennas includes: (1) the von Koch curve, (2) the Minkowski curve, (3) the Hilbert curve, (4) the Fractal tree, (5) the Sierpinski (gasket and carpet) Fractals, and (6) the Cantor set. These 
geometries also can be used in order to create EBG structures. However, only the Fractal shapes that are used for multiband antennas are able to form broader bandgap EBG. Actually, when an EBG structure has multiband, the broadband can be done by overlapping the different single-band to each other. In literature, Sierpinski (gasket and carpet) structures are widely used to create multi-band antenna [10]. From the idea of this design that is produced a hexagonal EBG with Fractal geometry, due to the characteristic of an equilateral triangle, Sierpinski gasket is chosen as a metal patch on the top surface in order to create the hexagonal EBG.

In this paper, the advantage of the novel hexagonal EBG structure was found by investigating in case of changing the gap between two adjacent Sierpinski triangles inside EBG unit cell. As a result, two structures are proposed; in which one structure introduces broad bandwidth and the others introduce dual bandgap. The design of EBG structures involving the mode-2 Sierpinski Gasket triangles (see Figure 1) and without via-holes or multilayer substrate is presented. The proposed EBG structures were simulated and measured using the method of suspended microstrip, which is recommended in [11] and a comparison between the measured results and simulated results has been carried out.

The rest of this paper is organized as follows. In Section 2, the detailed designs of the novel EBG structures are presented. By using the suspending microstrip method, the scatter parameters of the proposed EBG structure based on Sierpinski triangle that is formed by different steps, are characterized and simulated in Section 3. Besides, the bandgap of the conventional EBG structure is also determined for verifying the broader bandwidth of the proposed EBG structure. Next, the measurement of the proposed EBG structures in fabrication and discussions are also utilized in Section 4, while the conclusion is provided in Section 5.

\section{Hexagonal EBGs Design}

The resonance frequency and the bandwidth of an EBG structure depend on the unit-cell geometry together with substrate's relative dielectric permittivity and thickness. Each unit cell implements a distributed parallel network having one or more resonant frequencies. The resonance frequency of a parallel circuit is defined as follows:

$$
f_{C}=\frac{1}{2 \pi \sqrt{L C}} .
$$

The bandwidth is also an important consideration and is given by

$$
B W=\frac{1}{\eta} \sqrt{\frac{L}{C}},
$$

where $\eta$ is the free space impedance, which is $120 \pi$.

The designed unit cell geometry exhibits six symmetry planes, which makes it polarization-angle independent. The proposed EBG structures are shown in Figure 2, which are printed on an FR4 dielectric slab with dielectric constant of 4.4 and thickness of $1.6 \mathrm{~mm}$ and loss tangent of 0.02 . Dark

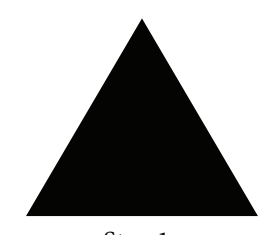

Step 1

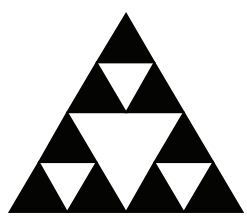

Step 3

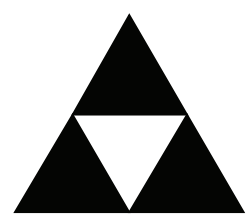

Step 2

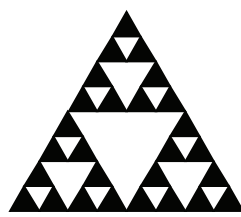

Step 4
FIgURE 1: Four steps to form a mode-2 Sierpinski gasket structure.

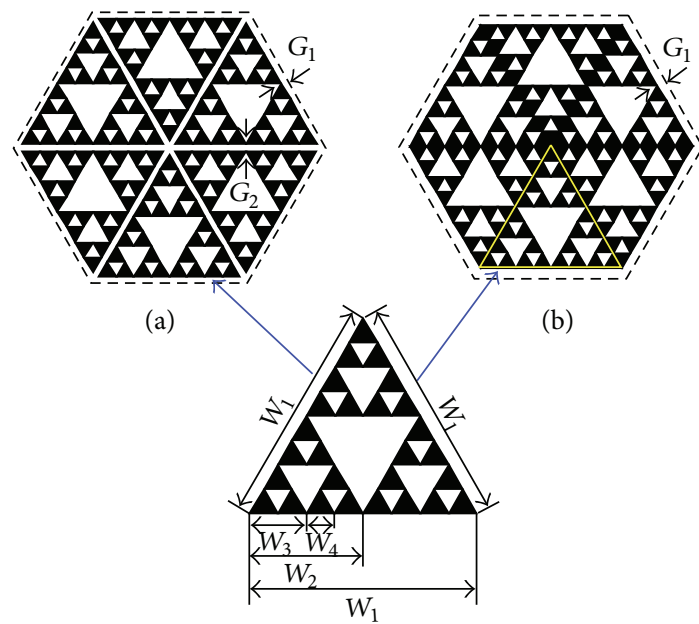

(c)

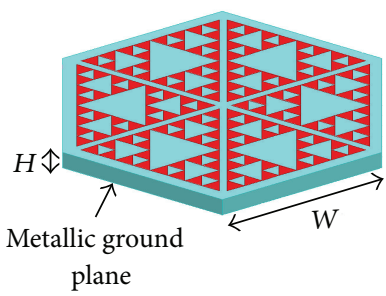

(d)

FIGURE 2: Unit cell geometries: (a) BEBG, (b) DEBG, (c) detailed Sierpinski gasket triangles, and (d) slide view of BEBG. Detailed dimensions: $W_{4}=W_{1} / 8 ; W_{3}=W_{1} / 4 ; W_{2}=W_{1} / 2 ; W_{1}=W-2\left(G_{2}+\right.$ $\left.G_{1}\right) / \sqrt{3}, G_{2}=0.5 \mathrm{~mm}, G_{1}=1 \mathrm{~mm}$.

parts in this figure represent the metallic periodic structure, which is etched on a dielectric substrate. Unit cell dimension is $W=10 \mathrm{~mm}$, and the metallization thickness is $18 \mu \mathrm{m}$.

The Sierpinski gasket is a well-known Fractal. The way it can be constructed and its main properties can be found in [12-14]. In [15], it is shown that the Sierpinski gasket is a special case of a wider class of Fractals that can be derived from the well-known Pascal's triangle. This class of Fractals can be derived in the following way. Consider an equiangular triangular grid whose rows shall be labelled by $n=1,2,3, \ldots$. Each row contains $n$ nodes, and to each node a number 


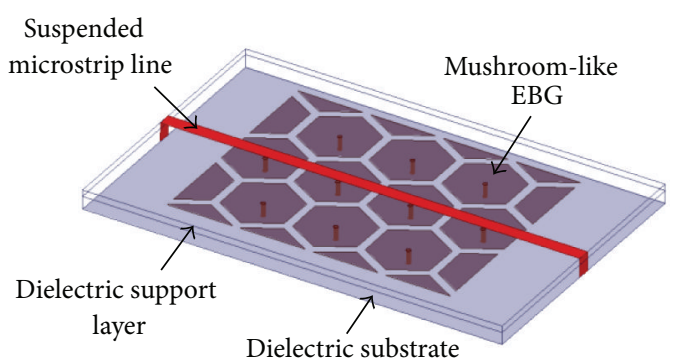

(a)

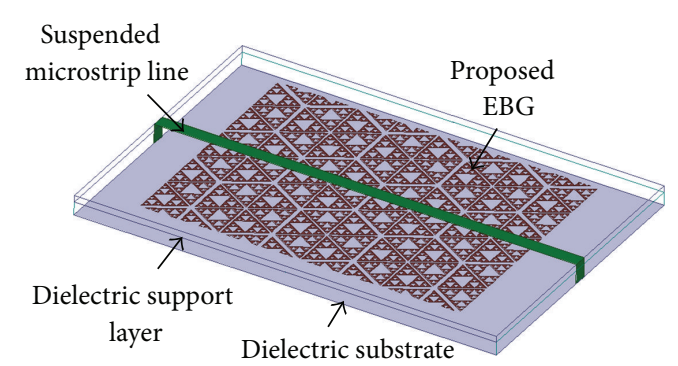

(b)

FIGURE 3: Geometry of $3 \times 4$ EBG arrays: (a) mushroom-like EBG array, and (b) proposed EBG array.

is attached. This number is the coefficient of the binomial expansion of $(x+y)^{n-1}$. Now delete from this grid those nodes that are attached to numbers that are divisible by $p$, where $p$ is a prime number. The result is a self-similar Fractal that will be referred to as the mod - $p$ Sierpinski gasket [13].

In this paper, The Sierpinski gasket triangle is formed by the following process (see Figure 2). Firstly, create an equilateral triangle patch with length edge of $W_{1}$. The second step of this Fractal EBG is constructed by etching out the center equilateral triangle of length edge $W_{2}$ inside the patch and subtracting it from the patch. The next stage is achieved by subtracting additional three equilateral triangles of length edge $W_{3}$ of the center triangles that follows the same technique adopted to realize the mode-2 Sierpinski gasket geometry (see Figure 2(c)).

From this design, the Sierpinski gasket triangles are arranged to repeat each $60^{\circ}$. The aim of this work allows modifying the resonance frequencies and the EBG operation bandwidth by changing the width and the gap $G_{1}$ between the hexagonal lattices. Moreover, by changing the gap $G_{2}$ between Sierpinski triangle units, two EBG structures are formed separately. The first one, which is called broadband EBG structure (BEBG), has a broader bandgap with the value of $G_{2}$ greater than $0 \mathrm{~mm}$. The second one, which is called dual-band EBG structure (DEBG), has dual bandgap when the value of $G_{2}$ is set at $0 \mathrm{~mm}$. These structures are shown in Figures 2(a) and 2(b), respectively.

\section{The Bandgap Characteristics}

In this section, the bandgap characteristics of two hexagonal EBG structures, which are formed by the Sierpinski triangle at $G_{2}=0$ and $G_{2}=0.5 \mathrm{~mm}$, are investigated. Besides, the bandgap of the conventional mushroom-like EBG is also determined in comparison with the proposed EBG structures. In order to analyze the bandgap properties of these EBG structures with finite unit number, an experiment concerning transmission through the above structures has been carried out. A $3 \times 4$ conventional EBG structure and proposed EBG structures have been simulated using the method of suspended microstrip, which is proposed by Fan to measure the bandgap characteristic of the EBGs, as shown in Figure 3 [11]. The operating frequency is set at $7 \mathrm{GHz}$, and the $50 \Omega$ microstrip line is placed on a dielectric support layer with the thickness of $0.5 \mathrm{~mm}$. The bandgap bandwidth will be defined with $S_{11}$ above $-5 \mathrm{~dB}$ and $S_{21}$ below $-30 \mathrm{~dB}$ at the same time.

3.1. EBG Structures at Different Steps. The hexagonal EBG structures with Sierpinski triangles at different steps are investigated in case the value of $W$ is fixed at $10 \mathrm{~mm}$ while the value of $G_{2}$ is set at $0 \mathrm{~mm}$ and $0.5 \mathrm{~mm}$. The simulation results of the EBG structures based on the Sierpinski triangle at step 1, step 2, and step 3 are shown in Figures 4, 5, and 6, respectively. In case of step 1, as can be seen in Figure 4(a), the EBG structure introduces a bandgap, which is ranging from $5.07 \mathrm{GHz}$ to $7.58 \mathrm{GHz}$, as $G_{2}$ is equal to $0.5 \mathrm{~mm}$. However, the bandgap was not found in case of $G_{2}$ value is equal to $0 \mathrm{~mm}$ (see Figure 4(b)). As can be seen from Figure 5(a), the EBG structure based on the Sierpinski triangle at step 2 has a lower bandgap than the one in the case of step 1 . The bandwidth of this bandgap covers from 4.22 to $6.88 \mathrm{GHz}$. Two bandgaps are defined in Figure 5(b) when the value of $G_{2}$ is set at $0 \mathrm{~mm}$. The lower bandgap covers from $2.25 \mathrm{GHz}$ to $2.96 \mathrm{GHz}$, and the higher one spans from $4.14 \mathrm{GHz}$ to $5.34 \mathrm{GHz}$. In this case, both bandgaps are defined not clearly, because the ranges of frequencies of $S_{11}$, which are up to $-5 \mathrm{~dB}$, are not flat. As can be obversed from Figure 6(a), the bandgap of EBG structure in step 3 is larger than the ones in the case of step 2 as $G_{2}$ is set at $0.5 \mathrm{~mm}$. This bandgap spans the frequencies from 4.32 to $7.92 \mathrm{GHz}$. Moreover, when $G_{2}$ is equal to $0 \mathrm{~mm}$ the bandgaps are defined easier than the one in the case of step 2. From Figure 6(b), the curve of S11 is quite flat in two bandgaps. The first bandgap spans the frequencies from 2.15 to $3.02 \mathrm{GHz}$; the second one has the bandwidth that is covered from $3.81 \mathrm{GHz}$ to $5.20 \mathrm{GHz}$. Next, the bandgap properties of the EBG structures at step 4 are considered by investigating the effect of parameters such as the size of unit cell $W$, and the gap between two adjacent unit cells $G_{1}$ to the bandgaps in both cases of DEBG and BEBG.

3.2. Broadband $E B G(B E B G)$. In general, in order to obtain a wider EBG operation bandwidth, it is necessary to increase $L$ and reduce $C$. Equivalent inductance $L$ can be increased using a thicker dielectric substrate and also including in the geometry narrow and long strips (lines). Equivalent capacitance $C$ can be reduced by reducing substrate's relative dielectric permittivity and increasing the gap between the metallization edge and the unit cell edge (and so the gap 


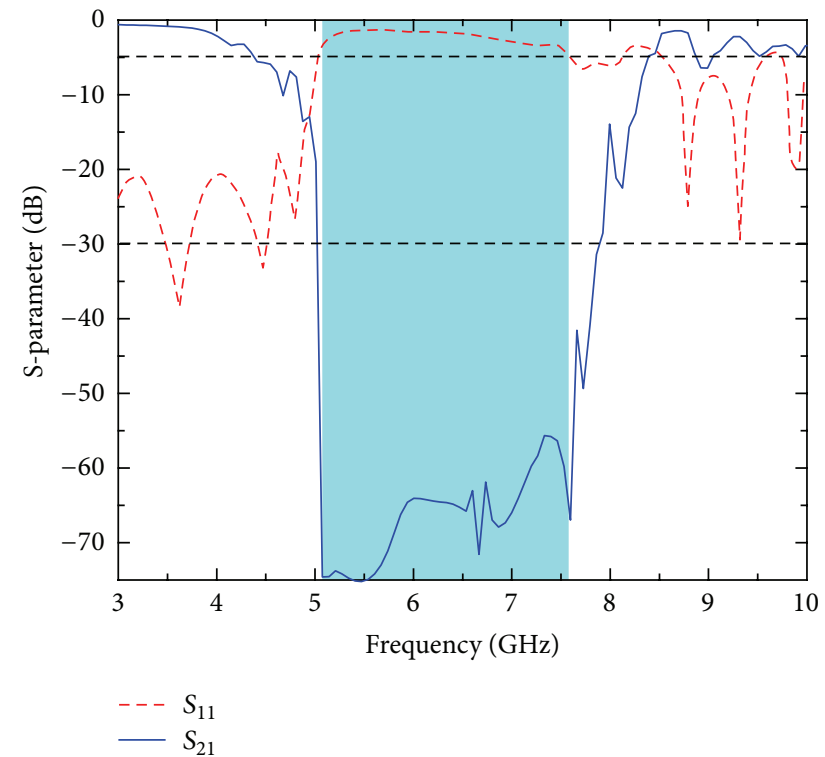

(a)

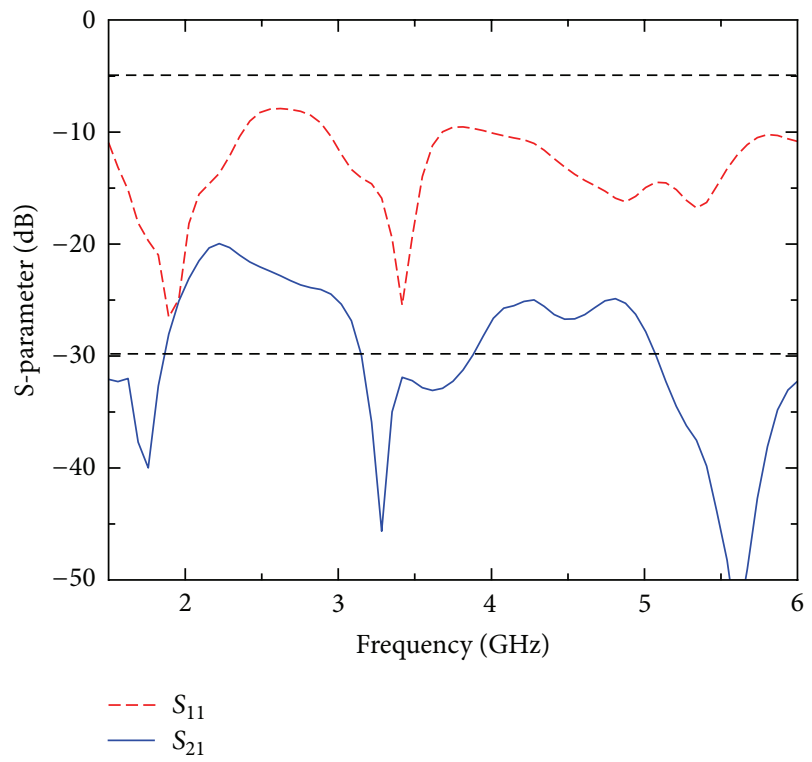

(b)

FIgURE 4: Operation bandwidth of hexagonal EBG structure based on Sierpinski triangle at step 1: (a) $G_{2}=0.5 \mathrm{~mm}$ and (b) $G_{2}=0 \mathrm{~mm}$.

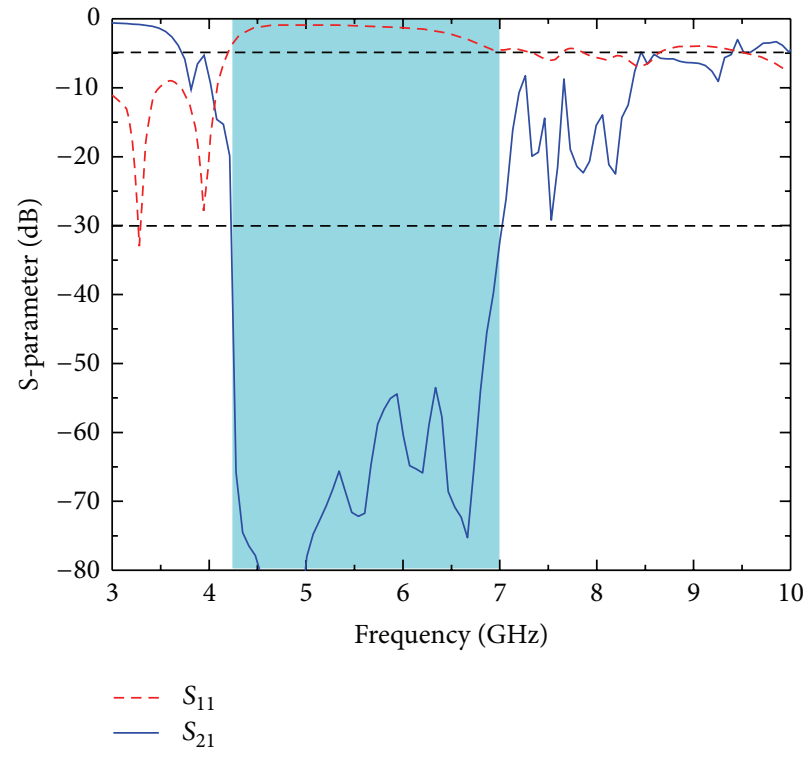

(a)

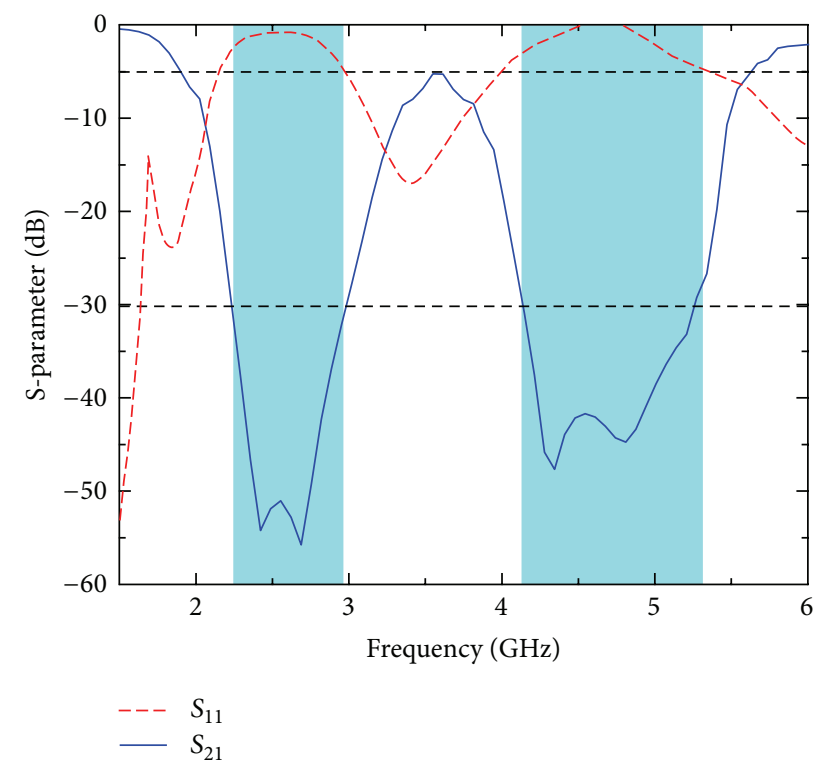

(b)

FIGURE 5: Operation bandwidth of hexagonal EBG structure based on Sierpinski triangle at step 2: (a) $G_{2}=0.5 \mathrm{~mm}$ and (b) $G_{2}=0 \mathrm{~mm}$.

between adjacent unit cells). Moreover, the thickness of substrate has to be adopted in order to obtain both compact size and broad EBG operation bandwidth.

In the EBG design procedure, if the dielectric material and its thickness have been chosen in this analysis, the total equivalent inductance $L$ cannot be altered. Therefore, only capacitance $C$ can be changed. Regarding the total equivalent $C$ of the unit cell, the contribution due to the gap between adjacent EBG cells (coplanar $C$ ) varies as the distance between unit cells reduces (which increases $C$ ) and also the distance between the Sierpinski triangles in the EBG unit cells (which decreases coplanar C). More important, in addition, the contribution due to the capacitance between unit-cell metallization and the unit-cell ground plane (parallel plates) is reduced for smaller unit-cell size $(W)$.

Finally, the wider EBG operation bandwidth for smaller unit-cell size $(W)$ of this novel design is due to more significantly decreased $C$ values of the parallel equivalent circuit. Moreover, the EBG operation bandwidth is also increased by reducing the relative dielectric permittivity for a given unitcell size. 


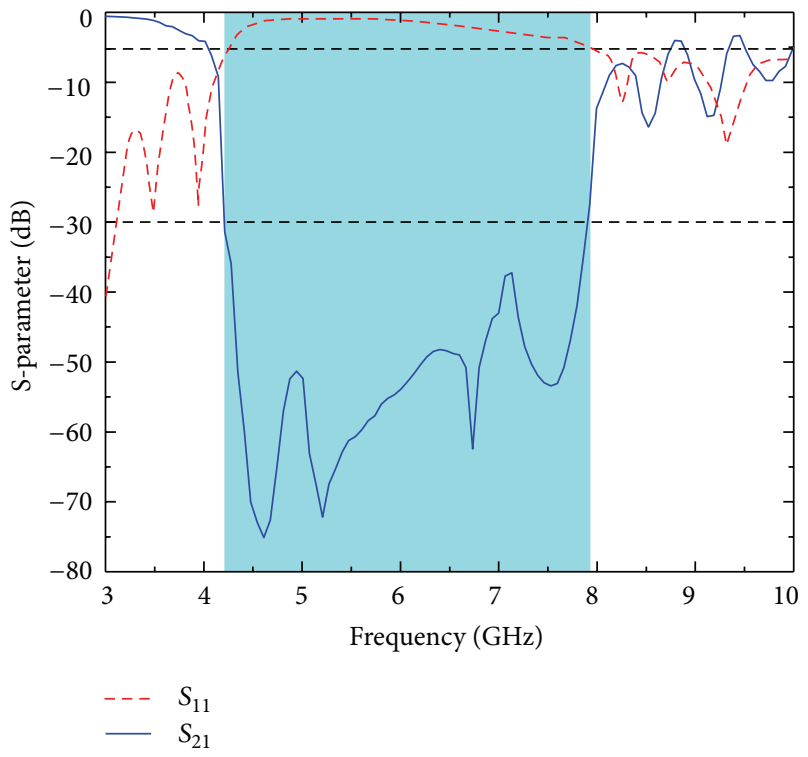

(a)

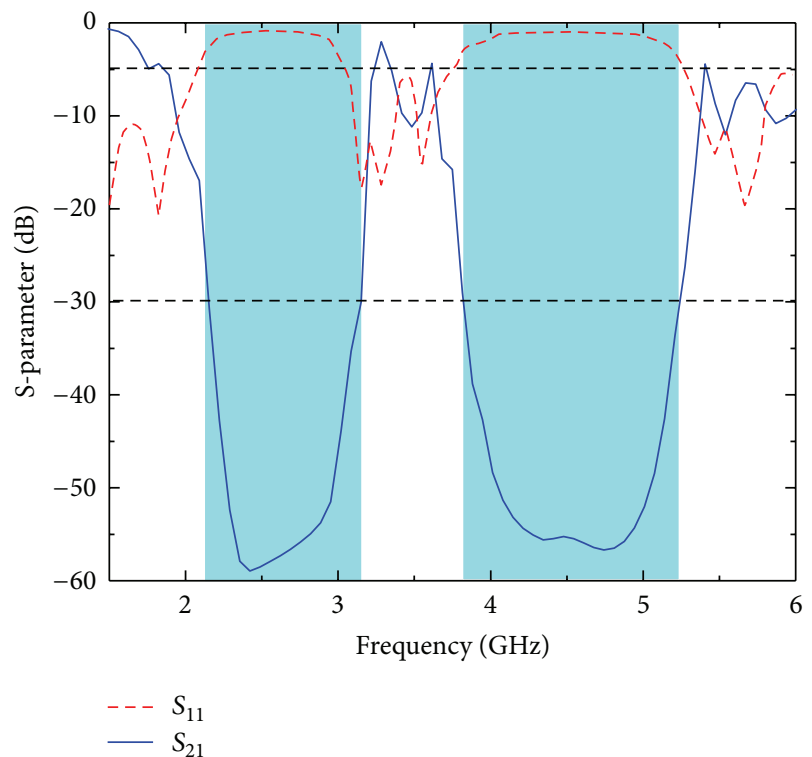

(b)

FIGURE 6: Operation bandwidth of Hexagonal EBG structure based on Sierpinski triangle at step 3: (a) $G_{2}=0.5 \mathrm{~mm}$ and (b) $G_{2}=0 \mathrm{~mm}$.

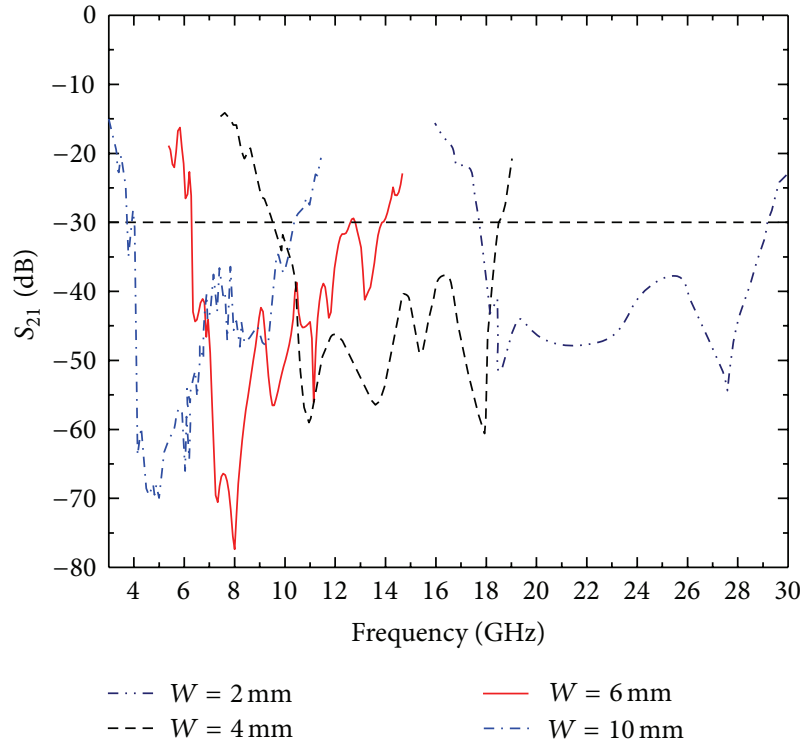

FIGURE 7: EBG operation bandwidth variations versus EBG unit-cell size $(W)$.

In order to analyses the effect of the parameters of dimension on the total equivalent capacitance $C$, the unitcell $(W)$ and the gap between adjacent EBG units $\left(G_{1}\right)$ are investigated while other parameters are constant. The unitcell size $(W)$ is very important parameter to take effect on the bandgap, which affects the equivalent capacitance of the LC resonant circuit of the EBG structure. Firstly, we investigate the bandgap of the EBG structure with different values of $W$. The unit cell size is increased from $2 \mathrm{~mm}$ to $10 \mathrm{~mm}$ while other parameters of the EBG structure are unchanged. The bandgap moves to lower frequency region with patch

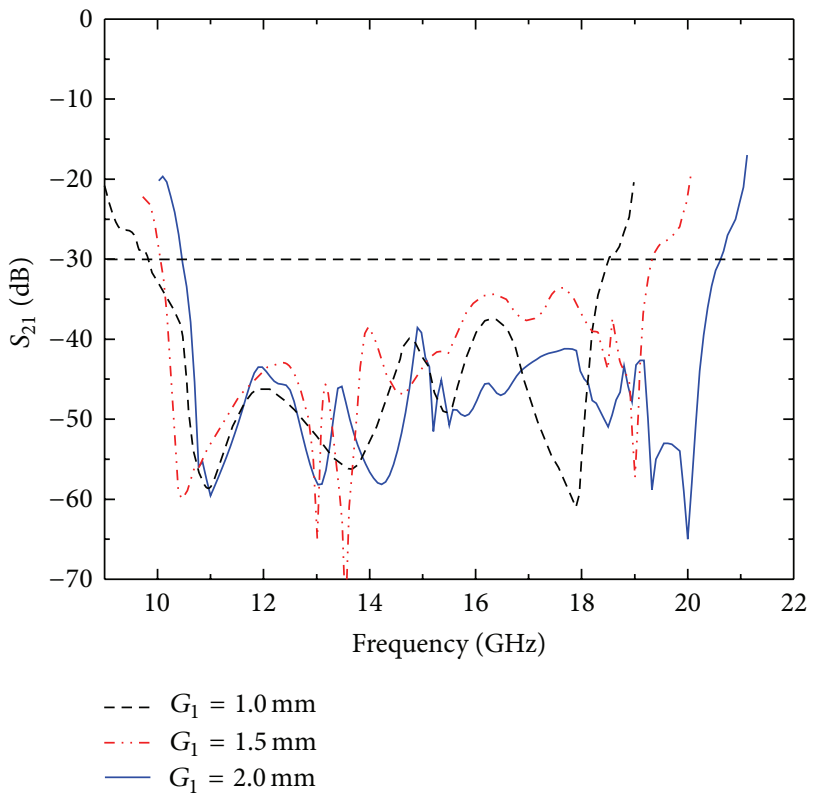

FIGURE 8: BEBG operation bandwidth variations versus the gap between adjacent EBG cells $\left(G_{1}\right)$ while the unit-cell $(W)$ is fixed at $4 \mathrm{~mm}$.

size increasing, and the bandgap becomes narrow. From Figure 7, we can see that the equivalent capacitance diminished when the patch size increases, so the resonant frequency is decreased. Since the bandgap width is proportional to $L / C$, the bandgap becomes narrow. The detail of this is shown in Table 1 .

Moreover, the effect of distance between adjacent EBG cells $\left(G_{1}\right)$ on the width of the bandgap is also investigated, and the simulated results are shown in Figure 8. As can 


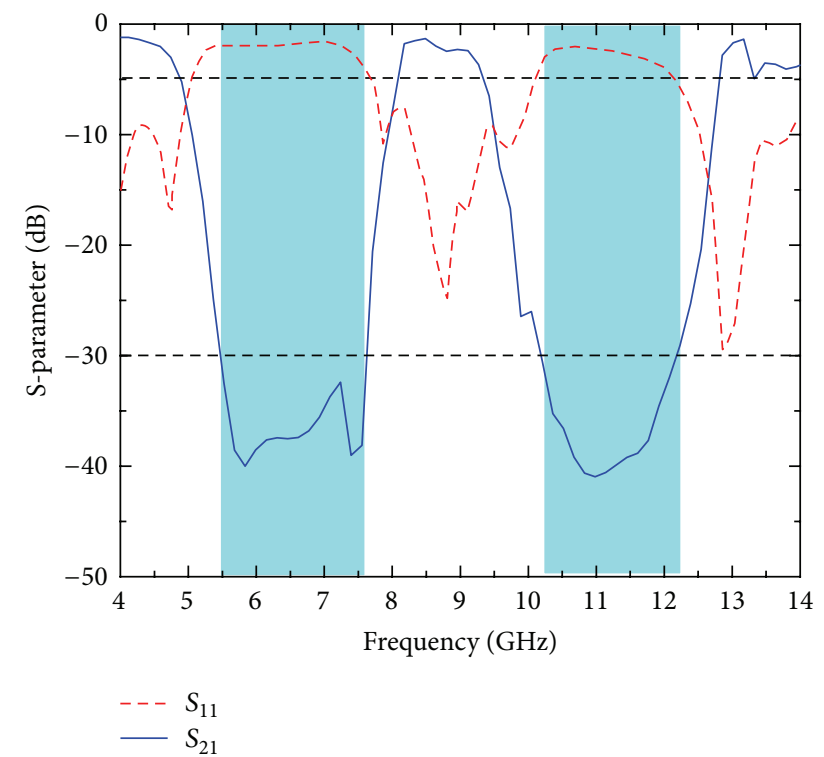

Figure 9: DEBG operation bandwidth with $G_{2}$ is equal to $0 \mathrm{~mm}$ and $W$ is equal to $4 \mathrm{~mm}$ while the other parameters are fixed.

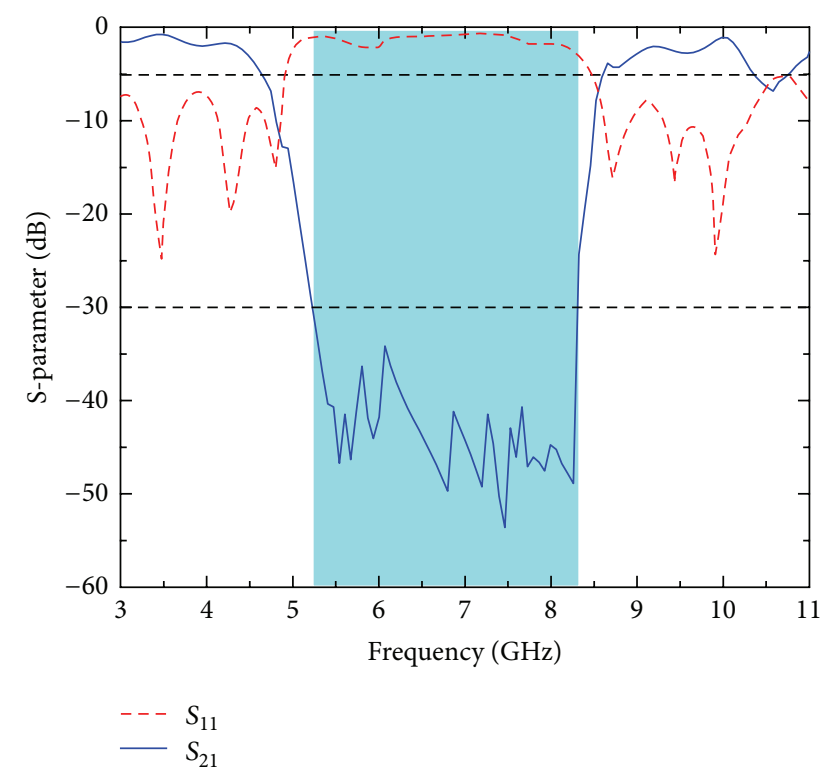

FIgURE 10: Simulated results of scatter parameters of conventional mushroom-like EBG structure $(W=10 \mathrm{~mm})$.

be observed from Figure 10, the EBG operation bandwidth becomes wider by increasing the gap $G_{1}$ between adjacent EBG cells and the bandgap moves to the higher frequency area. It is shown that the coplanar $C$ is increased with the higher values of $G_{1}$.

3.3. Dual-Band $E B G$ (DEBG). In this section, the gap $G_{2}$ between two adjacent Sierpinski triangles inside the EBG unit cell is also investigated while other parameters are constant. From Figure 9, it can be observes that two bandgaps are determined by transmission coefficient. The unique characteristic
TABLe 1: Parametric analysis of EBG unit cell.

\begin{tabular}{cccccc}
\hline Reference & $\begin{array}{c}\text { Unit cell } \\
\text { size } \\
(\mathrm{mm})\end{array}$ & $\begin{array}{c}\text { Thickness } \\
(\mathrm{mm})\end{array}$ & $\varepsilon_{r}$ & $\begin{array}{c}\text { Bandwidth } \\
(\%)\end{array}$ & $\begin{array}{c}\text { Resonance } \\
\text { frequency } \\
(\mathrm{GHz})\end{array}$ \\
\hline \multirow{5}{*}{ This paper } & $2(\lambda / 6.4)$ & $1.6(\lambda / 8)$ & 4.4 & 49 & 23.50 \\
& $4(\lambda / 5.2)$ & $1.6(\lambda / 13)$ & 4.4 & 65 & 14.05 \\
& $6(\lambda / 4.9)$ & $1.6(\lambda / 5)$ & 4.4 & 76 & 10.06 \\
& $10(\lambda / 4.0)$ & $1.6(\lambda / 4)$ & 4.4 & 87 & 7.20 \\
\hline
\end{tabular}

TABLE 2: Relative operation bandwidth of EBG structure for various $W$ while $G_{2}$ is Fixed at $0 \mathrm{~mm}$ and $0.5 \mathrm{~mm}$.

\begin{tabular}{lccc}
\hline $\begin{array}{l}\text { Unit cell size } \\
W(\mathrm{~mm})\end{array}$ & $\begin{array}{c}\mathrm{G}_{2}=0.5 \mathrm{~mm} \\
\text { Bandgap } \\
(\mathrm{GHz})\end{array}$ & $\begin{array}{c}\text { Bandgap 1 } \\
(\mathrm{GHz})\end{array}$ & $\begin{array}{c}\mathrm{G}_{2}=0 \mathrm{~mm} \\
\text { Bandgap 2 } \\
(\mathrm{GHz})\end{array}$ \\
\hline 2 & $17.74-29.25$ & $10.77-14.47$ & $18.80-21.95$ \\
4 & $9.48-18.62$ & $5.51-7.73$ & $10.20-12.33$ \\
6 & $6.23-13.88$ & $3.72-5.07$ & $6.72-8.53$ \\
10 & $4.05-10.35$ & $2.06-3.08$ & $3.71-5.26$ \\
\hline
\end{tabular}

TABLE 3: Operation bandwidth of proposed EBG structures with $W=10 \mathrm{~mm}$.

\begin{tabular}{|c|c|c|c|}
\hline \multirow[b]{2}{*}{ Methods } & \multirow{2}{*}{$\begin{array}{c}\text { BEBG } \\
\left(G_{2}=0.5 \mathrm{~mm}\right) \\
\text { Bandgap }(\mathrm{GHz}) \\
\text { (bandwidth }(\%))\end{array}$} & \multicolumn{2}{|c|}{$\operatorname{DEBG}\left(G_{2}=0 \mathrm{~mm}\right)$} \\
\hline & & $\begin{array}{c}\text { Bandgap 1 } \\
(\mathrm{GHz}) \\
\text { (bandwidth (\%)) }\end{array}$ & $\begin{array}{c}\text { Bandgap } 2 \\
(\mathrm{GHz}) \\
\text { (bandwidth (\%)) }\end{array}$ \\
\hline Simulated & $\begin{array}{c}4.05-10.35 \\
\quad(87)\end{array}$ & $\begin{array}{c}2.06-3.08 \\
(40)\end{array}$ & $\begin{array}{c}3.71-5.26 \\
(35)\end{array}$ \\
\hline Measured & $\begin{array}{c}4.15-10.00 \\
\quad(83)\end{array}$ & $\begin{array}{c}2.17-2.97 \\
(32)\end{array}$ & $\begin{array}{c}3.58-5.32 \\
(39)\end{array}$ \\
\hline
\end{tabular}

of this design that is the structure can be transformed the bandwidth of broadband into dual bandgap when $G_{2}$ is equal to zero. When the unit cell size $W$ is set at $4 \mathrm{~mm}$, the first bandgap spans from $5.51 \mathrm{GHz}$ to $7.73 \mathrm{GHz}$. The bandwidth of the second one is spreading from $10.20 \mathrm{GHz}$ to $12.18 \mathrm{GHz}$. This can be indicated more clearly in Table 2 .

It can be observed from Table 2 that these bandgaps move forward to the lower frequency regions. It is clear that when the value of $G_{2}$ is up to zero, two above bandgaps are moving to overlap each other and the broader bandgap can be obtained.

3.4. Conventional Mushroom-Like EBG. In order to verify the bandgap characteristic of the broadband EBG structure (BEBG), an array of $3 \times 4$ unit cells of hexagonal mushroomlike EBG is investigated (see Figure 3(a)). This EBG is also constructed on a dielectric substrate with a relative permittivity of 4.4 and thickness of $1.6 \mathrm{~mm}$. The unit cell dimension is fixed at $10 \mathrm{~mm}$. The simulated result of transmission coefficient is shown in Figure 10. The bandgap is center at $6.77 \mathrm{GHz}$, and the bandgap bandwidth is from $5.22 \mathrm{GHz}$ to $8.32 \mathrm{GHz}$, achieve the bandgap bandwidth about $46 \%$. As can be observed from Table 1, the bandgap bandwidth of 


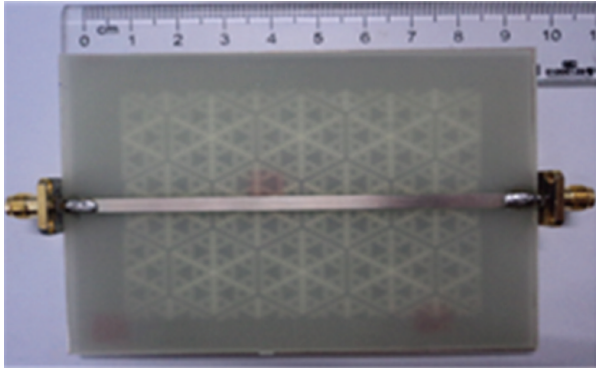

(a)

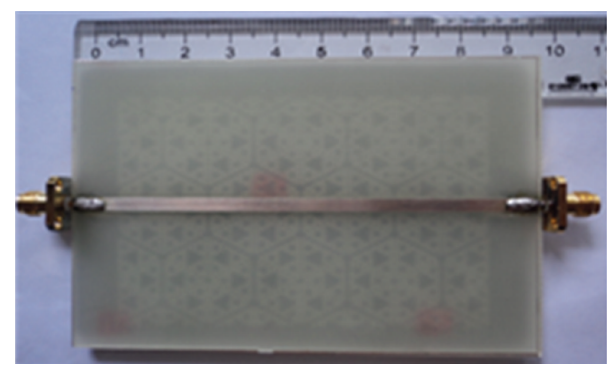

(b)

FIGURE 11: Photos of the proposed structures with suspended microstrip: (a) array of $3 \times 4$ DEBG cells and (b) array of $3 \times 4$ BEBG cells.

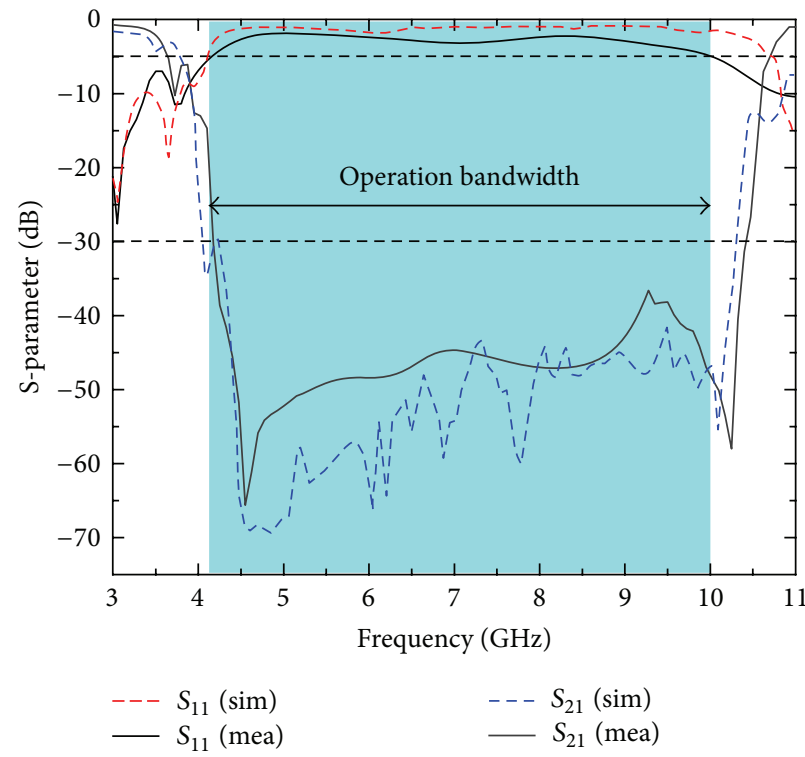

(a)

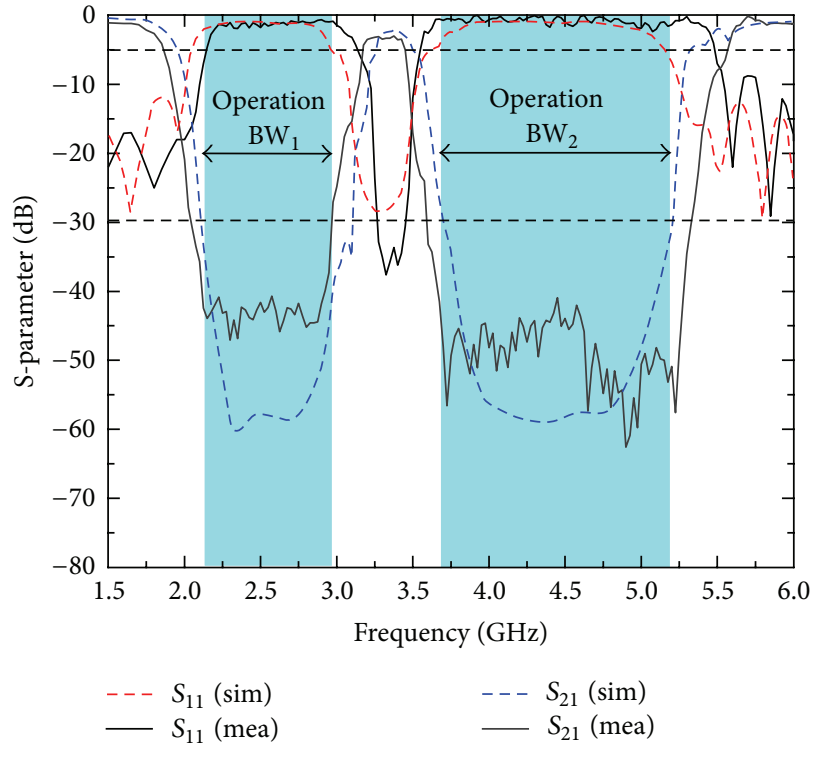

(b)

Figure 12: EBG operation bandwidth $(W=10 \mathrm{~mm})$ : (a) BEBG and (b) DEBG.

the BEBG structure at $W=10 \mathrm{~mm}$ is larger than that of the conventional EBG at the same dimension of the unit cell.

\section{Results and Discussions}

Both schematics of proposed EBG structures were simulated and taken into comparison with the measurement of the fabrication. The microstrip lines are soldered with SMA connector to measure the scatter parameters. The measured results of scatter parameters of the arrays are performed by Anritsu 37369D network analyzer. Photos of the proposed EBG structures are shown in Figure 11. The simulated and the measured results of the parameters of BEBG structure and DEBG structure are shown in Figures 12(a) and 12(b), respectively. It is very efficient to determine the bandgap by the transmission curves. From the measurement of $S_{11}$ with the criteria of $-5 \mathrm{~dB}$, the operation bandwidth can be determined easily when the transmission coefficient $S_{21}$ is below $-30 \mathrm{~dB}$. As can be observed from Figure 12(a), the BEBG introduces a bandgap between the frequencies $4.15 \mathrm{GHz}-10 \mathrm{GHz}$ with the criteria of $-30 \mathrm{~dB}$, from that the electromagnetic wave cannot propagate. The bandgap bandwidth is about $83 \%$. The reason is that the surface impedance of the EBG structure becomes very high within the bandgap. From Figure 12(b), two distinct bandgaps of DEBG structure are defined in which the first bandgap is ranging from 2.17 to $2.97 \mathrm{GHz}$ and the bandwidth of the second bandgap is spreading from 3.58 to $5.32 \mathrm{GHz}$. The bandgap bandwidths of the DEBG structure are $32 \%$ and $39 \%$, respectively. It can be observed from Table 3 that the measured results show good agreement with the simulated results.

\section{Conclusion}

A novel EBG design is presented using Sierpinski gasket triangles, which are arranged to repeat $60^{\circ}$ to form the hexagonal EBG unit cell. Two EBG structures, which have broadband and dual bandgap, are proposed by setting the value of the gap between two adjacent Sierpinski inside the unit cell is equal to $0 \mathrm{~mm}$ and greater than $0 \mathrm{~mm}$, respectively. 
The suspending microstrip method is used to simulate the scatter coefficients of the EBG structure. The bandwidth of BEBG structure is much larger than that of the conventional EBG. The results show a good agreement between simulations and measurements. Due to several advantages of the structures such as using inexpensive dielectric substrate FR4 and planar structure, the EBG structures are promising for low profile and low cost antennas in broadband or multiband applications.

\section{References}

[1] F. R. Yang, K. P. Ma, M. Yongxi Qian, and T. Itoh, "A uniplanar compact photonic-bandgap (UC-PBG) structure and its applications for microwave circuits," IEEE Transactions on Microwave Theory and Techniques, vol. 47, no. 8, pp. 1509-1514, 1999.

[2] J. D. Joannopoulos, R. D. Meade, and J. N. Winn, Photonic Crystals, Princeton University Press, Princeton, NJ, USA, 1995.

[3] E. Yablonovitch, "Photonic band-gap structure," Journal of the Optical Society of America B, vol. 10, no. 2, pp. 283-295, 1993.

[4] D. Sievenpiper, L. Zhang, R. F. Jimenez Broas, N. G. Alexöpolous, and E. Yablonovitch, "High-impedance electromagnetic surfaces with a forbidden frequency band," IEEE Transactions on Microwave Theory and Techniques, vol. 47, no. 11, pp. 2059-2074, 1999.

[5] A. Stark, S. Prorok, and A. F. Jacob, "Broadband EBG structures with compact unit cell," in Proceedings of the 38th European Microwave Conference, EuMC 2008, pp. 698-701, Amsterdam, The Netherlands, October 2008.

[6] L. Liang, C. H. Liang, X. W. Zhao, and Z. J. Su, "A novel broadband EBG using multi-period mushroom-like structure," in Proceedings of the International Conference on Microwave and Millimeter Wave Technology (ICMMT '08), pp. 1609-1612, Nanjing, China, April 2008.

[7] R. Coccioli, F. R. Yang, K. P. Ma, and T. Itoh, "Aperture-coupled patch antenna on UC-PBG substrate," IEEE Transactions on Microwave Theory and Techniques, vol. 47, no. 11, pp. 2123-2130, 1999.

[8] M. Rahman and M. A. Stuchly, "Circularly polarised patch antenna with periodic structure," IEE Proceedings: Microwaves, Antennas and Propagation, vol. 149, no. 3, pp. 141-146, 2002.

[9] M. F. Abedin, M. Z. Azad, and M. Ali, "Wideband smaller unit-cell planar EBG structure and their application," IEEE Transactions on Antennas and Propagation, vol. 56, no. 3, pp. 903-908, 2008.

[10] W. J. Krzysztofik, "Modified Sierpinski fractal monopole for ISM-bands handset applications," IEEE Transactions on Antennas and Propagation, vol. 57, no. 3, pp. 606-615, 2009.

[11] M. Y. Fan, R. Hu, Q. Hao, X. X. Zhang, and Z. H. Feng, "New method for 2D-EBG structures research," Hongwai Yu Haomibo Xuebao/Journal of Infrared and Millimeter Waves, vol. 22, no. 2, pp. 127-131, 2003.

[12] C. Puente, J. Romeu, and A. Cardama, "Fractal-shaped antennas," in Frontiers in Electromagnetics, D. H. Werner and R. Mittra, Eds., pp. 48-93, IEEE Press, Piscataway, NJ, USA, 2000.

[13] C. Puente-Baliarda, J. Romeu, R. Pous, and A. Cardama, "On the behavior of the sierpinski multiband fractal antenna," IEEE Transactions on Antennas and Propagation, vol. 46, no. 4, pp. 517-524, 1998.
[14] H. O. Peitgen, H. Jurgens, and D. Saupe, Chaos and Fractals, New Frontiers in Science, Springer, New York, 1992.

[15] N. S. Holter, A. Lakhtakia, V. K. Varadan, V. V. Varadan, and R. Messier, "On a new class of planar fractals: the Pascal-Sierpinski gaskets," Journal of Physics A, vol. 19, no. 9, article 047, pp. 17531759, 1986. 

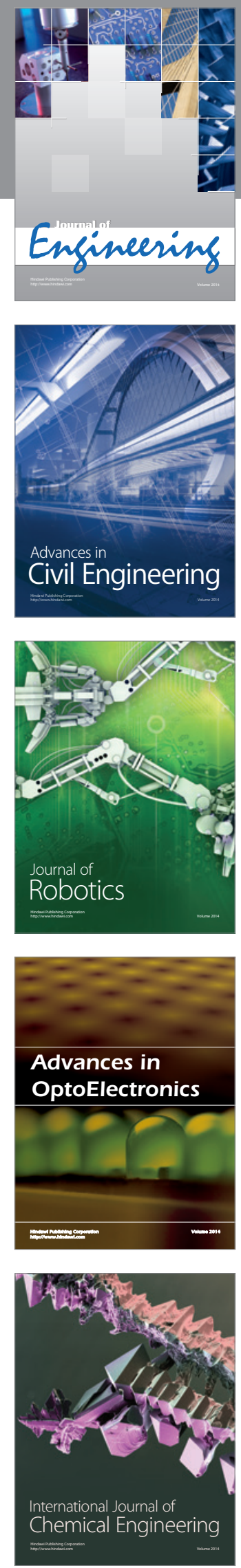

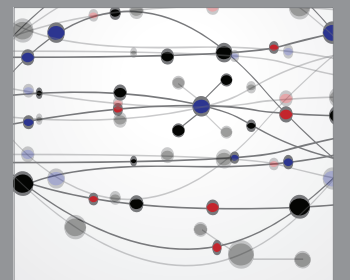

The Scientific World Journal
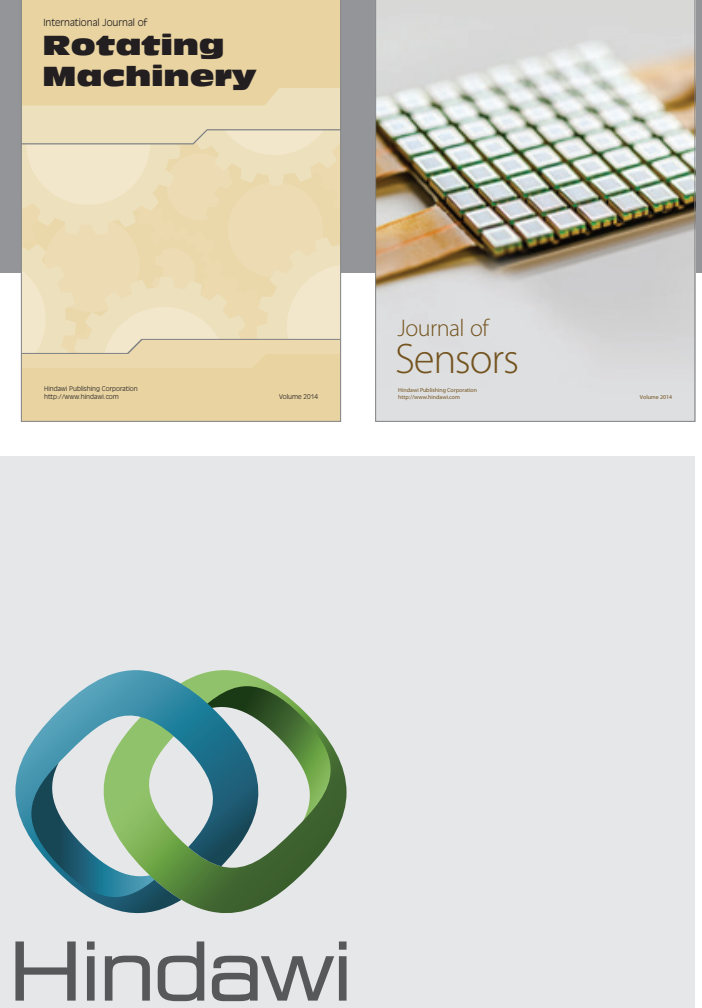

Submit your manuscripts at http://www.hindawi.com
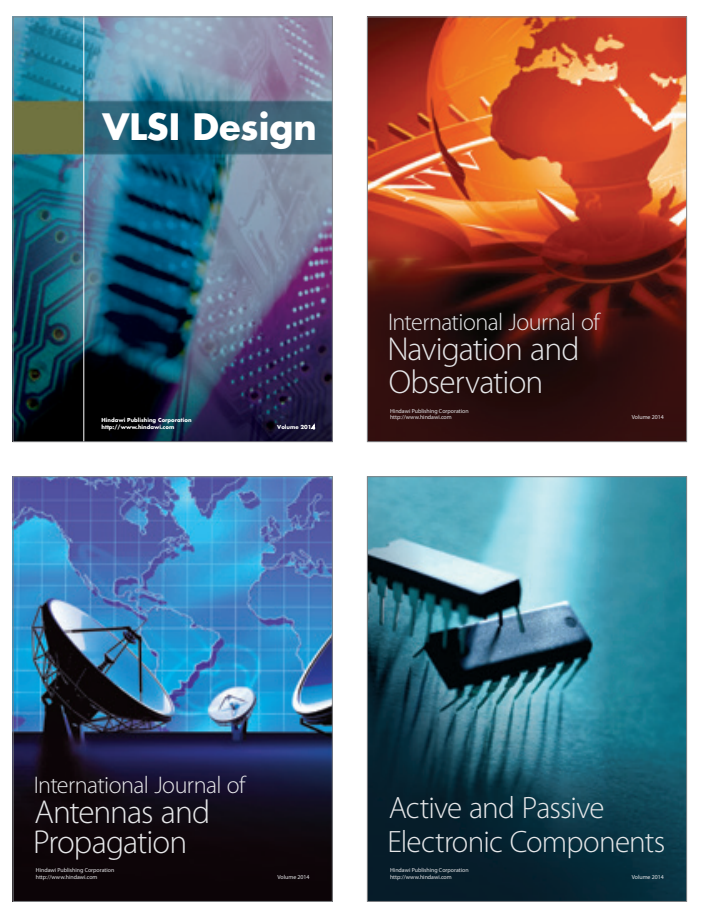
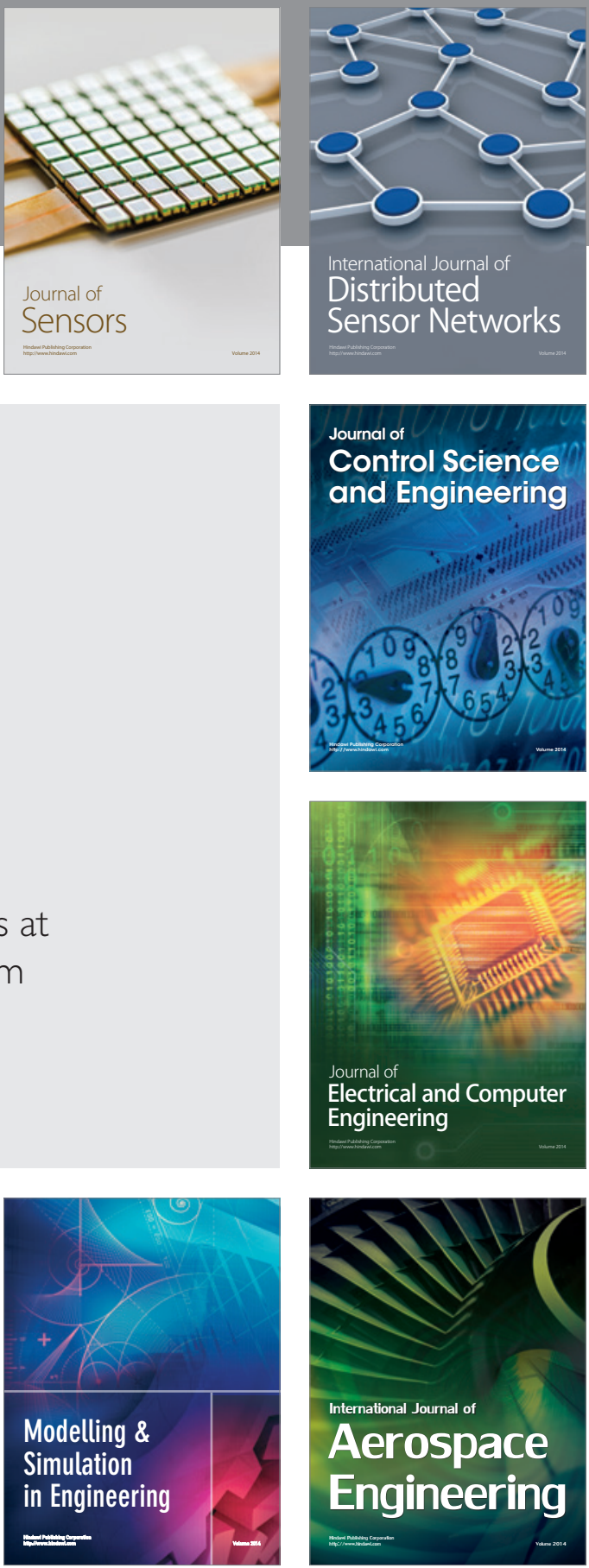

Journal of

Control Science

and Engineering
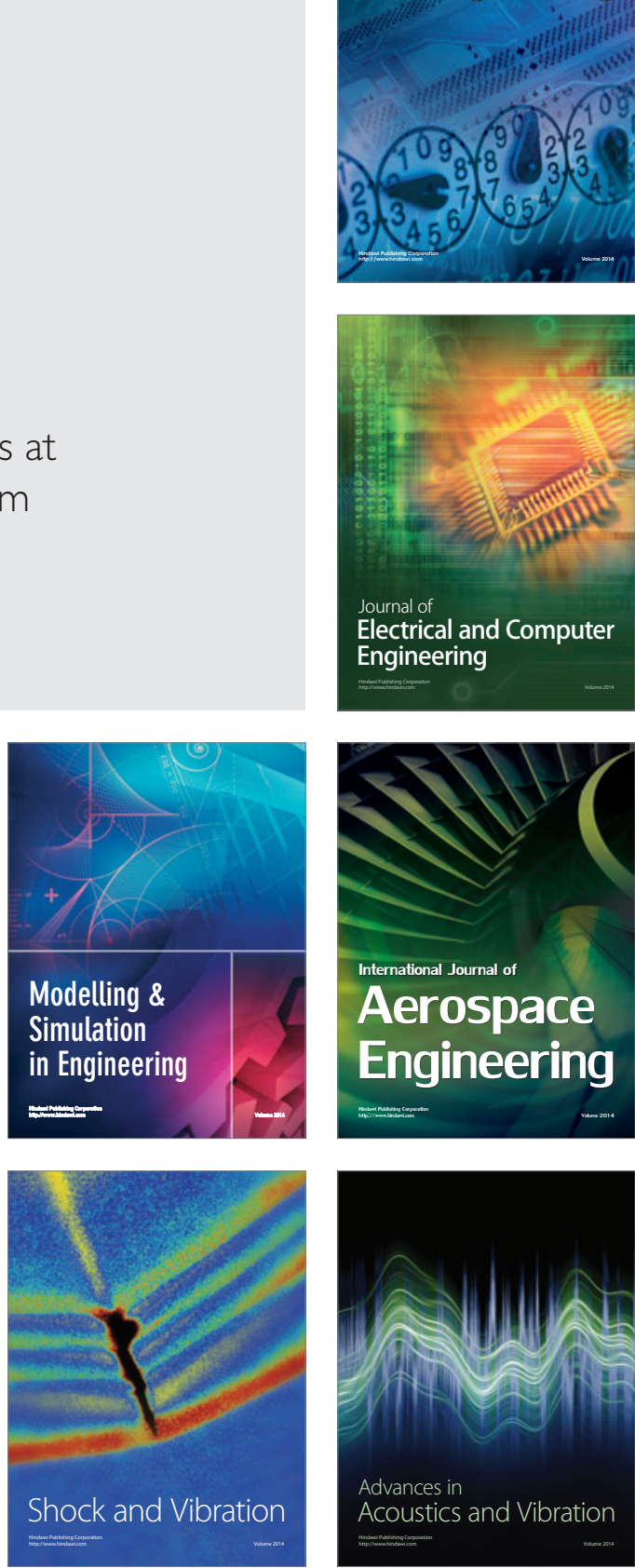